\title{
Enactive Approaches to Designing and Understanding Immersive Interactive Virtual Environments in Mental Healthcare
}

\author{
Tor Alexander Bruce \\ Northumbria University \\ CoCreate, School of Design \\ Newcastle upon Tyne \\ UK \\ tor.a.bruce@northumbria.ac.uk
}

\begin{abstract}
Recent work demonstrates the potential benefits of narrative techniques for individuals with traumas that underpin many mental health problems. Likewise, research over the last decade highlights possibilities for immersive technologies in providing ways for clinicians and patients to benefit from the delivery of therapeutic interventions. However, while immersive technologies are maturing to the point of being easily configurable in a range of spaces, evidence is lacking on their efficacy and integration into healthcare settings. This research aims to understand how immersive interactive virtual environments (IIVEs), as viewed through an enactivist lens, could provide opportunities for healthcare providers to incorporate narrative techniques into mental health interventions.
\end{abstract}

Immersive Interactive Virtual Environments; Enactivism; Human Computer Interaction; Mental Health; Narrative Techniques; Convivial Tools; Co-Design; Generative Exercises

\section{INTRODUCTION}

This research explores the role of immersive interactive virtual environments (IIVEs) in the context of mental health therapeutic interventions. For the purpose of this research, immersive technologies are recognised as a particular type of Virtual Reality (VR) technology that are broadly understood across a range of devices, tools, applications and settings (Onyesolu, Ezeani and Okonkwo, 2012). The study will focus on a specific class of immersive technology, IIVEs, which in layman-terms are rooms containing projectors with ability to represent and simulate different realities. In the case of the technology being used in this research, the projections are mapped onto three surrounding walls, with the projections being a setting or a set of visuals or sounds, as defined by participants. The walls may or may not have touch interactive sensors and this type of technology differs from the immersive head-mounted display, which is an immersive device worn by the individual.

The research is funded by Immersive Interactive, a leading global provider of IIVEs, forming part of an industrial collaboration together with Alliance Psychological Services, a company who deliver services involving therapeutic interventions with up to 10,000 mental health patients per year. It is envisioned that the work will contribute knowledge at the intersection of human-computer interaction and mental health research in three ways: (i) through the development and testing of a generative toolkit for co-designing IIVEs with mental health experts, clinical staff and people living with mental health trauma; (ii) the design and evaluation of new enactive approaches in IIVEs that support narrative-based interventions for people living with mental health trauma; and; (iii) to build on new theoretical understandings of IIVE design, underpinned by enactivist ontology. The research is being guided by the following initial research questions:

(i) How do expert senior mental health workers, clinicians and experts by experience who have presented past trauma, interpret (generative) tools used to imagine how an immersive virtual environment could function?

(ii) What type of features could immersive interactive virtual environments provide in helping people reproduce the conditions to support their understanding of life events? 


\section{HCI AND MENTAL HEALTH}

Research has demonstrated the potential benefits of narrative techniques for individuals with traumas that underpin many mental health problems, with narrative therapy being recognised as a postmodern approach placing clients as experts of their own lives and of the stories they relay (Guindon, 2011). Aponte and Patrick describe the 'chaotic and incoherent life narrative' of individuals who have experienced mental health traumas and discuss 'externalizing', as a means of a person discovering ways to identify with their problems outside of themselves (2017:5) Likewise, research over the last decade has highlighted possibilities for immersive technologies in providing new ways for clinicians and patients to benefit from the delivery of new therapeutic interventions.

In their agenda-setting work on mental health and wellbeing, Thieme et al. suggest that human computer interaction $(\mathrm{HCl})$ should adopt a more holistic approach in expanding its focus, stating: 'The [HCl] field needs to increase its effort to include strategies that can positively contribute to people's mental wellbeing in both the design and evaluation of future technology' (2015:6). Focused more on the broad class of technologies addressed in this research, Maples-Keller et al. discuss effectiveness of incorporating a wide range of psychiatric disorders in VR settings with a specific focus on developing exposure-based interventions for anxiety. They report on uses of such technology for people with autism, schizophrenia, psychosis, PTSD, anxiety and panic disorder amongst others, noting:

The first study using virtual reality to treat a psychological disorder was published in 1995 [by Rothbaum et al.]... Virtual reality has emerged as a viable tool to help in a number of disorders...meta-analyses have indicated that $V R$ is an efficacious tool, compares favourably to comparison conditions, and has lasting effects that generalize to the real world (2017:14).

Despite this, there is still a very limited understanding of how such technologies might be meaningfully incorporated into more personalised care provision and how clinicians and patients might be supported in adopting and creating content for them. As such, while immersive technologies are maturing to the point of being easily configurable in a wide range of spaces, including hospitals, schools and care units, there exists a lack of evidence on their efficacy and integration into healthcare settings. Aguilera suggests that 'there are many opportunities and immense potential for digital technologies to improve health and mental health service provision but there are many challenges to overcome as well' (2015:6). As such, while O'Leary et al. consider how $\mathrm{HCl}$ is: 'well-positioned to design technologies for and with mental health peer supporters to create tools that enhance collaborative strategies to address the unmet need for mental health support' (2017:1470), there are ongoing barriers and userfacing issues to making this a reality in the context of a more robust mental health service delivery.

\section{A GENERATIVE DESIGN APPROACH}

This research expands on prior work by aiming to understand how a specific form of immersive technology, IIVEs, could provide opportunities for healthcare providers to incorporate narrative techniques into therapeutic interventions. In doing so, the research aims to enable clinicians to personalise content and promote greater control for patients in terms of how they experience therapy. To explore this, the research will engage in a generative design approach (Sanders and Stappers, 2012) where IIVEs will be co-designed with clinicians and patients. Das et al. consider:

\begin{abstract}
A generative session is a meeting in which users do generative exercises; the participants are given tools such as illustrations, post-it notes, pictures, or, sets of expressive components, in order to create artefacts that express their thoughts, feelings, and ideas. For instance, this process can involve that the participants are given a "toolkit"... (2015:3)
\end{abstract}

Following Das and Sanders and Stappers, studies will be initially conducted using a bespoke 'convivial toolkit' (Sanders and Stappers, 2012) that will support idea generation and dialogue with healthcare experts and experts by experience. Through the introduction of tools (Figure 1) and carefully scaffolded discussion, it is expected the research will develop new understandings of the ways immersive spaces might be utilised in this healthcare context.

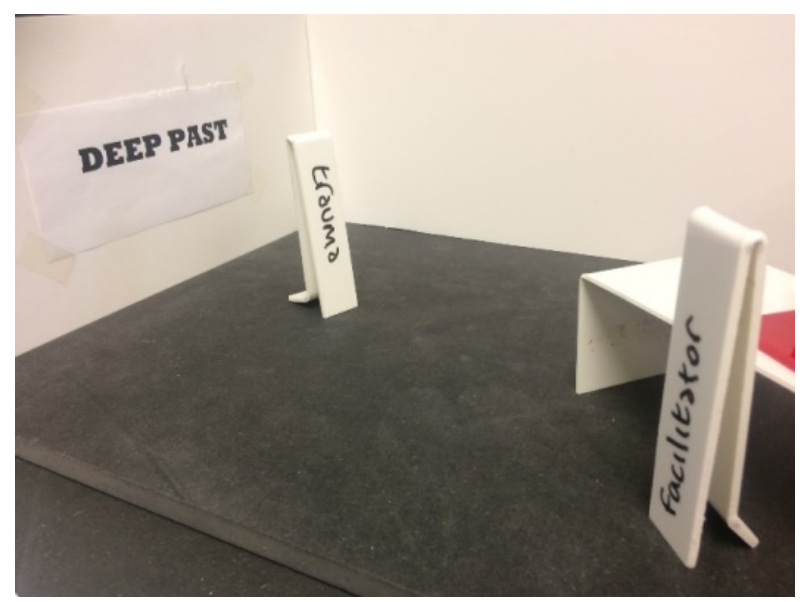

Figure 1: Acrylic figures and prototype small-scale immersive room 
Through these tools the study will examine ways people with complex mental health problems can be supported in sharing narratives of their life course; by moving through a digitally augmented space and interacting with rich media related to particular epochs of their lives.

\section{AN ENACTIVIST BRIDGE}

To underpin a research quest which seeks ultimately to position human participants in a virtual environment, enactivist theories (as a further extension of theories of embodiment with roots in phenomenology) are drawn upon. Enactivism provides a philosophical framework for understanding interrelations between human mind, body and space which takes on specific significance in immersive environments. The theory of embodiment, or "embodied cognition" (Bandura, 1994; Clark, 1999; Dourish, 2004; Rocha, 2012; Kirsh, 2013; Jasanoff, 2019) supports the notion that some features of cognition are shaped by aspects of the entire body of the organism, in that a combination of brain and body influence one another as part of a whole system of self. Here, enaction becomes a concept (used in one way, as well as other related ideas), to describe how the human organism's "cognitive system of self" interlinks autonomously with its environment (Figure 2) via a process of coupling $^{1}$, through sensorimotor $^{2}$ activity.

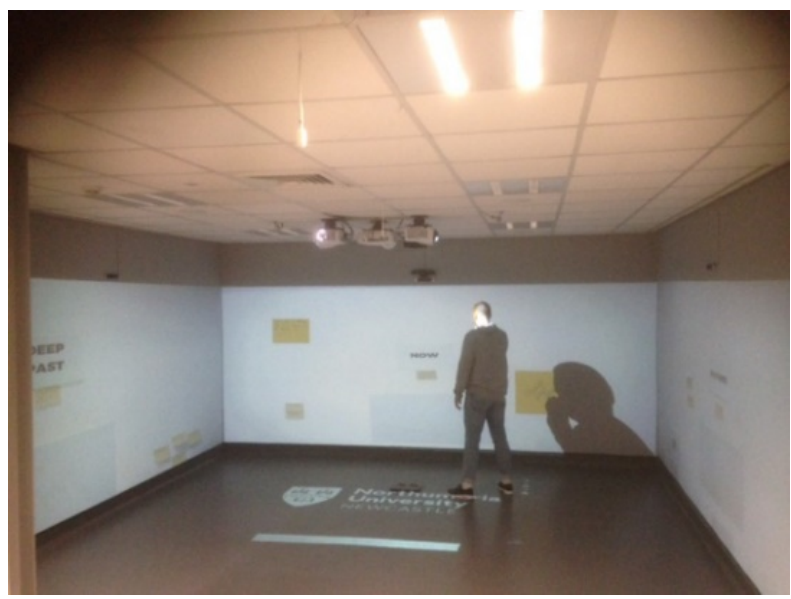

Figure 2: Immersive Interactive Virtual Environment containing trial-simulation process and actor

Building on the work of Varela, Thompson and Rosch who proposed 'as a name the term enactive' (1991:9), Barandiaran supports that enactivism is both: 'maturing and diversifying as a theoretical framework' (2017:409). Enactivist theory argues that cognition arises via a dynamic interaction

\footnotetext{
${ }^{1}$ The act of joining two things together

2 Sensorimotor relates to the senses and motor movements of the human system
}

between an acting organism (a human agent) and its environment and 'emphasises emergent cognitive structures that self-organise as a result of interaction between organism and environment' (Ward, Silverman, Villalobos, 2017:368).

People who participate in immersive interactive environments have opportunity to engage with their bodies, in a standing [not seated] approach which could offer potential to consider the conceptual mind, body and the immersive space as intrinsically linked. Jasanoff notes that 'even if we tacitly admit' to the importance of brain, body and environment playing a co-joined role, then 'this in turn affects how we understand and treat a range of social and behavioural problems in the real world' (2018:164). Building on this view is Dourish who considers: 'Embodied interaction is not a technology or a set of rules. It is a perspective on the relationship between people and systems' (2004: 192). The enactivist ontology sustained throughout this research study will argue that cognition arises via a dynamic interaction between an acting organism (agent) and its environment. What this could lead to is a personalised approach, positioning each participant in an autonomous light where the immersive environment they become coupled to can enable human interaction with technology at a level of efficacy, in the context of becoming a potential recovery model (via an enactive approach) in mental healthcare.

\section{REFERENCES}

Aguilera, A. (2015) Digital Technology And Mental Health Interventions: Opportunities and Challenges. Volume 191, No. 771 [Online] Available at: https://escholarship.org/uc/item/0nj447nk

Aponte, D.A., Patrick, S. (2017) Narrative Approaches to Counseling Survivors of Child Sexual Abuse, Wisdom in Education, Volume 7: Issue 2, Article 2 [Online] Available at: https://scholarworks.lib.csusb.edu/cgi/viewconte nt.cgi?referer=https://www.google.com/\&httpsred ir $=1 \&$ article $=1059 \&$ context $=$ wie

Bandura, A. (1994) Self-efficacy. In V.S. Ramachaudran (Ed.) Encyclopedia of human behaviour (Vol. 4, pp. 71-81). New York: Academic Press. (Reprinted in $\mathrm{H}$. Friedman [Ed.]. Encyclopedia of mental health. San Diego: Academic Press, 1998) [Online] Available at: https://pdfs.semanticscholar.org/63c0/16b24e57 5bc19f58710a3ed49838878560f8.pdf

Barandiaran. X.E. (2017) Autonomy and Enactivism: Towards a Theory of Sensorimotor Autonomous Agency, Topoi 36, 409-430 [Online] Available at: DOI: 10.1007/s11245-016-9365-4 
Clark, A. (1999) Being There. Putting Brain, Body, and World Together Again, Massachusetts Institute of Technology, MIT Press, United States of America

Das, A., Bøthun, S., Reitan, J., Dahl, Y. (2015) The Use of Generative Techniques in Co-design of mHealth Technology and Healthcare Services for COPD Patients. International Conference of Design, User-Experience and Usability. DUXU 2015 [Online] Available at: DOI:10.1007/978-3319-20889-3_54

Dourish, P. (2004) Where the Action is. The Foundations of Embodied Interaction. MIT Press, Massachusetts Institute of Technology, Massachussetts

Guindon, M.H. (2011) A counselling primer. An introduction to the profession. Routledge, New York

Jasanoff, A. (2018) The Biological Mind, Basic Books, New York

Kirsh, D., (2013) Embodied Cognition and the Magical Future of Interactive Design. ACM Trans. On Human Computer Interaction, Vol. 20, pp. 1, 4. [Online] Available at: https://quote.ucsd.edu/cogs1/files/2012/09/KirshFinal-acm_reading.pdf

Maples-Keller, J.L., Bunnell, Brian, E., Sae-Jin, K., Rothbaum, B.O. (2017) The use of virtual reality technology in the treatment of anxiety and other psychiatric disorders. Harv. Rev. Psychiatry. DOI: 10.1097/HRP.0000000000000138

O'Leary, K. et al. (2017) Design Opportunities for Mental Health Peer Support Technologies, Proceedings of the 2017 ACM Conference on Computer Supported Cooperative Work and
Social Computing, CSCW '17. 1470-1484 [Online] Available at: https://dl.acm.org/doi/pdf/10.1145/2998181.2998 349?download=true

Onyesolu, M.O., Ezeani, I., Obikwelu, R.O. (2012) A Survey of Some Virtual Reality Tools and Resources. Intech Open [Online] Available at: https://pdfs.semanticscholar.org/3b76/cfd337b19 e73d6a19b03f696f41776217d94.pdf

Rocha, M. (2012) Cognitive, Embodied or Enacted? Contemporary Perspectives for $\mathrm{HCl}$ and Interaction. Transtechnology Research, Plymouth [Online] Available at: http://www.transtechresearch.net/wpcontent/uploads/2015/05/TTReader2011_012_R ocha-01.pdf

Sanders, E.-N and Stappers, P.J. (2012 [2018]) Convivial Toolbox: General Research For The Front End Of Design, BIS Publishers, The Netherlands

Thieme, A., Wallace, J., Meyer, T.D., Oliver, P. (2015) Designing for Mental Wellbeing: Towards a More Holistic Approach in the Treatment and Prevention of Mental Illness. ICPS Proceedings. British $\mathrm{HCl}$ '15 [Online] Available at: https://dl.acm.org/citation.cfm?doid=2783446.27 83586

Varela, F.J., Thompson, E., Rosch, E. (1991) The Embodied Mind. MIT Press. Massachusetts Institute of Technology, Cambridge, Massachusette; London, England

Ward, D., Silverman, D., Villalobos, M. (2017) Introduction: The Varieties of Enactivism. Springer Science-Business Media Dordrecht. Topoi 36, 365-375 [Online] Available at: DOI: $10.1007 / \mathrm{s} 11245-017-94$ 